\title{
A futuristic view of energy saving and related energy rebound effect in Bangladesh using complete decomposition model (CDM)
}

\author{
S. Khosruzzaman ${ }^{a}$, M. A. Asgar ${ }^{b}$, M. A. Asgarc ${ }^{c}$,K. M. R. Rahmand and S. Akbar \\ ${ }^{a}$ Institute of Natural Sciences, National University, Gazipur, Bangladesh., ${ }^{b}$ Uttara University, \\ Dhaka, Bangladesh ${ }^{c}$ East-West University, Dhaka, Bangladesh ${ }^{d}$ Bangladesh Atomic Energy \\ Commission, Dhaka, Bangladesh
}

\begin{abstract}
In this paper Complete Decomposition Model is used to compute the future energy saving pattern from the difference of the 'trend' and 'real' values of energy consumption. The 'trend' is defined as a sum of activity effect and the energy use in the base year. The 'real' is defined as a sum of energy consumption in the base year and the change in energy consumption due to the activity effect, structural effect and intensity effect. This analysis is carried out in respect of Bangladesh for the period 2008-2030. The economic sectors that are taken in to account are agriculture, industry and service. The futuristic view shows that Bangladesh can save about 47.47 MTOE in agriculture sector and 34.96 MTOE from service sector. On the other hand, industry sector, which is accounted for $58 \%$ of the total energy consumption, failed to save energy, rather the country consumed 227 MTOE more energy than usual. The energy rebound effect that relies upon the activity effect and structural effect has also been estimated to examine the energy uses pattern of these sectors. The aggregate energy rebound effect was found to be 1480 MTOE, of which activity effect and structural effect contribute $91.21 \%$ and $8.78 \%$ respectively.
\end{abstract}

Key Words: Complete Decomposition Method, Energy saving, Energy rebound effect, Bangladesh.

\section{Introduction}

The decomposition method is an effective tool for energy demand analysis and also for energy and environmental description (Ang, 2000). This approach takes into account the relationship between energy consumption in different sectors of economic activity and energy-related economy. It gives a differential and quantities view of the implementation of energy conservation measures. The forefront study of the application of the decomposition of energy conservation was presented by others (Sun 2003). However, most of the studies were limited to two economic dimensions such as energy intensity and GDP. We have extended the method to analyze the energy saving of different economic sectors of Bangladesh projected over the period 2008-2030.

Energy saving reflects the effects of technological progress and structural changes of an economy. Energy saving indicates the total reduction of energy use if the overall economic activity remains unchanged. If the effectiveness of production technology increases, energy saving takes place. Again, if the share of a sector of the total production volume decreases, energy saving may also occur. Energy saving also takes into account the structural shift such as the shift towards the use of services instead of energy commodities (Punyong, 2008).

The energy rebound effect captures the development that takes place if technological change is not directly included. It is the calculation of a sector's response in terms of energy consumption to the development of the value added plus the structural effect. The energy rebound effect is a reflection of the indirect effect of technological development on energy use insofar as technological development increased economic growth accompanied as structural shift in the economy.

In this study, the three dimension complete decomposition model was formulated to analyze the energy saving and energy rebound effect of different sector in Bangladesh. The study analyzed data of the period 2007-2030, as an attempt to assess the extent of the acclaimed success in Bangladesh.

*Corresponding author. E-mail: skzaman1971@yahoo.com 


\section{Materials and Methods}

We have used the available up-to-date data from different national and international sources like Bangladesh Bureau of Statistics (BBS), Bangladesh Power Development Board (BPDB), Petrobangla, Asian Development Bank (ADB), and the World Bank (WB), etc. The annual data of Gross Domestic Product (GDP) is converted into US\$ at the rate of 2000. The GDP and commercial energy consumption of 2007 are considered as base values. In 2008, the GDP growth rate was $5.2 \%$ and it is considered at $10 \%$ in 2030 . This is the proposed futuristic goal of the present government as documented in election manifesto and expressed in vision 2021. The contribution of agriculture, industrial and service sector GDP are 22\%, 28\% and 50\% respectively in 2007 (BBS, 1992-2008). This figure will increase to $13 \%, 45 \%$ and $42 \%$ respectively in 2030 . In 2008 , the energy growth rate was $8 \%$ and it will rise to $9.5 \%$ in 2030 (BBS, 19922008). The contribution of commercial energy consumption in agriculture, industrial and service sector are 11\%, 46\% and 43\% respectively in 2007 (BBS, 1992-2008). This figure rose to $5 \%, 62 \%$ and $33 \%$ respectively in 2030 . The energy co-efficient was 1.53 in 2007 and this figure is estimated to be 0.95 in 2030 .

The Complete Decomposition Method was used to construct the energy saving model in different sector. The model starts with $G D P$-related energy intensity,

$E_{t}$ is the sum of sector's energy consumption $E_{i t}$ :

$\mathrm{E}_{\mathrm{t}}=\sum_{\mathrm{i}} \mathrm{E}_{\mathrm{it}}$

Where $\mathrm{i}$ is the index of sector.

The total energy consumption $\mathrm{E}_{\mathrm{t}}$ is a function of three variables:

1) LEVEL OF OUTPUT, $A_{t}$, which measures aggregate sectoral activity either in economic or physical units and consists of sectoral inputs:

$\mathrm{A}_{\mathrm{t}}=\sum_{\mathrm{i}} \mathrm{A}_{\mathrm{it}}$

2) ENERGY INTENSITY OF SECTORS, $I_{i t}$, defined as sectoral energy consumption $\mathrm{E}_{\mathrm{it}}$ per unit of activity $\mathrm{A}_{\mathrm{it}}$ :

$$
\mathrm{I}_{\mathrm{it}}=\mathrm{E}_{\mathrm{it}} / \mathrm{A}_{\mathrm{it}}
$$

3) STRUCTURAL PARAMETER, $\mathrm{S}_{\mathrm{it}}$, defining the share of sectors $i$ in the aggregate sectoral output in the year $t$ :
$\mathrm{S}_{\mathrm{it}}=\mathrm{A}_{\mathrm{it}} / \mathrm{A}_{\mathrm{t}}$

The following equations decompose total energy consumption into the terms of activity, structure and energy intensity:

$$
\begin{aligned}
E t & =\sum_{i}\left(A_{t} X S_{i t} X I_{i t}\right) \\
& =\sum_{i}\left(A_{t} X\left[A_{i t} / A_{t}\right] X\left[E_{i t} / A_{i t}\right]\right)
\end{aligned}
$$

In the decomposition approach, changes in energy consumption between the base year and year $t$ can be divided into activity, intensity and structure effects:

$$
\begin{aligned}
\Delta \mathrm{E}_{\mathrm{ot}} & =\mathrm{E}_{\mathrm{t}}-\mathrm{E}_{\mathrm{o}}-\mathrm{P}_{\mathrm{i}}\left(\mathrm{A}_{\mathrm{t}} \mathrm{X} \mathrm{S}_{\mathrm{it}}\right)-\sum_{\mathrm{i}}\left(\mathrm{A}_{\mathrm{o}} \mathrm{X} \mathrm{S}_{\mathrm{io}} \mathrm{XI}_{\mathrm{io}}\right) \\
& =\mathrm{GDP}_{\text {effect }}+\mathrm{S}_{\text {effect }}+\mathrm{I}_{\text {effect }}
\end{aligned}
$$

Where, GDP effect, $\mathrm{S}_{\text {effect }}$ and $\mathrm{I}_{\text {effect }}$ represents activity effect, structural effect and intensity effect respectively. Following the decomposition method (Sun. 1998, Sun. 2001) these three effects can be decomposed as below.

Activity effect $\left(G_{\text {effect }}\right)=\sum_{i}\left(\Delta \mathrm{A}_{\mathrm{t}} \mathrm{S}_{\text {io }} \mathrm{I}_{\text {io }}\right)+(1 / 2) \Sigma \Delta \mathrm{A}_{\mathrm{t}}\left(\mathrm{S}_{\text {io }}\right.$ $\left.\Delta \mathrm{I}_{\mathrm{it}}+\Delta \mathrm{S}_{\mathrm{it}} \mathrm{I}_{\mathrm{io}}\right)+(1 / 3) \sum_{\mathrm{i}}\left(\Delta \mathrm{A}_{\mathrm{t}} \Delta \mathrm{S}_{\mathrm{it}} \Delta \mathrm{I}_{\mathrm{it}}\right)$

Structural effect $\left(\mathrm{S}_{\text {effect }}\right)=\sum_{\mathrm{i}}\left(\mathrm{A}_{\mathrm{o}} \Delta \mathrm{S}_{\mathrm{it}} \mathrm{I}_{\mathrm{io}}\right)+(1 / 2) \sum_{\mathrm{i}} \Delta \mathrm{S}_{\mathrm{it}}\left(\mathrm{A}_{\mathrm{o}} \Delta \mathrm{I}_{\mathrm{it}}\right.$ $\left.+\Delta \mathrm{A}_{\mathrm{t}} \mathrm{I}_{\mathrm{io}}\right)+(1 / 3) \sum_{\mathrm{i}}\left(\Delta \mathrm{A}_{\mathrm{t}} \Delta \mathrm{S}_{\mathrm{it}}^{\mathrm{i}} \Delta \mathrm{I}_{\mathrm{it}}\right)$

And

Intensity effect $\left(\mathrm{I}_{\text {effect }}\right)=\sum_{\mathrm{i}}\left(\mathrm{A}_{\mathrm{o}} \mathrm{S}_{\text {io }} \Delta \mathrm{I}_{\mathrm{it}}\right)+(1 / 2) \sum_{\mathrm{i}} \Delta \mathrm{I}_{\text {it }}\left(\mathrm{A}_{\mathrm{o}} \Delta \mathrm{S}_{\text {it }}\right.$ $\left.+\Delta \mathrm{A}_{\mathrm{t}} \mathrm{S}_{\mathrm{io}}\right)+(1 / 3) \sum_{\mathrm{i}}\left(\Delta \mathrm{A}_{\mathrm{t}} \Delta \mathrm{S}_{\mathrm{it}} \Delta \mathrm{I}_{\mathrm{it}}\right)$

Here,

$\mathrm{E}_{\mathrm{t}}, \mathrm{E}_{0}=$ Total energy used in year $\mathrm{t}$ and 0 (base year)

$\mathrm{I}_{\mathrm{io}}+\Delta \mathrm{I}_{\mathrm{it}}, \mathrm{I}_{\mathrm{io}}=$ energy intensity of sector $\mathrm{i}$ in year $\mathrm{t}$ and 0 , respectively

$\mathrm{S}_{\mathrm{io}}+\Delta \mathrm{S}_{\mathrm{it}}, \mathrm{S}_{\mathrm{io}}=$ output share of sector $\mathrm{i}$ in year $\mathrm{t}$ and 0

$\mathrm{A}_{\mathrm{o}}+\Delta \mathrm{A}_{\mathrm{t}}, \mathrm{A}_{\mathrm{o}}=$ level of aggregated activity in year $\mathrm{t}$ and 0

$\Delta \mathrm{A}_{\mathrm{t}}=\mathrm{A}_{\mathrm{t}}-\mathrm{A}_{0}$

$\Delta \mathrm{S}_{\text {it }}=\mathrm{S}_{\text {it }}-\mathrm{S}_{\text {io }}$ and

$\Delta \mathrm{I}_{\text {it }}=\mathrm{I}_{\text {it }}-\mathrm{I}_{\text {io }}$

From equation (7), the 'real' energy consumption in the year $\mathrm{t}$ can be expressed as.

Real

$$
=\mathrm{E}_{\mathrm{t}}=\Delta \mathrm{E}_{\mathrm{ot}}+\mathrm{E}_{\mathrm{o}}
$$

The GDP effect is used to predict the 'trend' of the energy consumption in year $\mathrm{t}$ as in the following equation. 
Trend

$$
=\mathrm{GDP}_{\text {effect }}+\mathrm{E}_{\mathrm{o}}
$$

Energy saving is defined as the difference between Trend and Real. Thus,

$\psi=$ Real - Trend

$=\Delta \mathrm{E}_{\mathrm{ot}}+\mathrm{E}_{\mathrm{o}}-\mathrm{GDP}_{\text {effect }}-\mathrm{E}_{\mathrm{o}}$

$=\Delta \mathrm{E}_{\mathrm{ot}}-\mathrm{GDP}_{\text {effect }}$

$=\mathrm{GDP}_{\text {effect }}+\mathrm{S}_{\text {effect }}+\mathrm{I}_{\text {effect }}-\mathrm{GDP}_{\text {effect }}$

$=\mathrm{S}_{\text {effect }}+\mathrm{I}_{\text {effect }}$

Energy saving is achieved only if $\psi<0$, which indicates that the actual increase of energy consumption (real) is less than what should have, otherwise, resulted from the growth of the economy (trend). This condition implies that the energy consumption has been comparatively reduced (saved), which is the indicator of the success of the energy conservation plan. In contrast, if $\psi>0$, energy saving is not achievable.

The energy saving model $(\psi)$ can be written as,

$$
\begin{aligned}
& \psi=\mathrm{S}_{\text {effect }}+\mathrm{I}_{\text {effect }} \\
& =\sum_{\mathrm{i}}\left(\mathrm{A}_{\mathrm{o}} \Delta \mathrm{S}_{\mathrm{it}} \mathrm{I}_{\mathrm{io}}\right)+(1 / 2) \sum_{\mathrm{i}} \Delta \mathrm{S}_{\mathrm{it}}\left(\mathrm{A}_{\mathrm{o}} \Delta \mathrm{I}_{\mathrm{it}}+\Delta \mathrm{A}_{\mathrm{t}} \mathrm{I}_{\mathrm{io}}\right)+(1 / 3) \\
& \sum_{\mathrm{i}}\left(\Delta \mathrm{A}_{\mathrm{t}} \Delta \mathrm{S}_{\mathrm{it}} \Delta \mathrm{I}_{\mathrm{it}}\right)+\sum_{\mathrm{i}}\left(\mathrm{A}_{\mathrm{o}} \Delta \mathrm{S}_{\mathrm{io}} \Delta \mathrm{I}_{\mathrm{it}}\right)+(1 / 2) \sum_{\mathrm{i}} \Delta \mathrm{I}_{\mathrm{it}}\left(\mathrm{A}_{\mathrm{o}}\right. \\
& \left.\Delta \mathrm{S}_{\mathrm{it}}+\Delta \mathrm{A}_{\mathrm{t}} \Delta \mathrm{S}_{\mathrm{io}}\right)+(1 / 3) \sum_{\mathrm{i}}\left(\Delta \mathrm{A}_{\mathrm{t}} \Delta \mathrm{S}_{\mathrm{it}} \Delta \mathrm{I}_{\mathrm{it}}\right) \\
& =\sum_{\mathrm{i}}\left(\mathrm{A}_{\mathrm{o}} \Delta \mathrm{S}_{\mathrm{it}} \mathrm{I}_{\mathrm{io}}\right)+(1 / 2) \sum_{\mathrm{i}} \Delta \mathrm{S}_{\mathrm{it}}\left(\mathrm{A}_{\mathrm{o}} \Delta \mathrm{I}_{\mathrm{it}}+\Delta \mathrm{A}_{\mathrm{t}} \mathrm{I}_{\mathrm{io}}\right)+\sum_{\mathrm{i}}\left(\mathrm{A}_{\mathrm{o}} \mathrm{S}_{\mathrm{io}}\right. \\
& \left.\Delta \mathrm{I}_{\mathrm{it}}\right)+(1 / 2) \sum_{\mathrm{i}} \Delta \mathrm{I}_{\mathrm{it}}\left(\mathrm{A}_{\mathrm{o}} \Delta \mathrm{S}_{\mathrm{it}}+\Delta \mathrm{A}_{\mathrm{t}} \mathrm{S}_{\mathrm{io}}\right)+(2 / 3) \sum_{\mathrm{i}}\left(\Delta \mathrm{A}_{\mathrm{t}} \Delta \mathrm{S}_{\mathrm{it}}\right. \\
& \left.\Delta \mathrm{I}_{\mathrm{it}}\right)
\end{aligned}
$$

Energy saving appears mathematically in these models as a negative value of $\psi$. Thus the negative values have $S_{\text {effect }}$ and I effect, represent the saving caused by the change of the respective dimensions.

Malaska et al. in 1999 proposed a group of metrics in order to relate the decomposition analysis to matters of sustainability. Dematerialization of energy production, immaterialization of consumption and rebound effect are important factors in shaping sustainable energy. We have analyzed the energy rebound effects of different sectors based upon Malaska's approach (Malaska et al., 1999).

The equation for energy sustainability $(E s)$ can be presented in the following matrix form

$$
E S=\left(\begin{array}{l}
E_{D e} \\
E_{S a} \\
E_{\mathrm{Re}}
\end{array}\right)=\left(\begin{array}{l}
-1+0+0 \\
-1-1+0 \\
+0+1+1
\end{array}\right)\left(\begin{array}{l}
I_{\text {effect }} \\
S_{\text {effect }} \\
G D P_{\text {effect }}
\end{array}\right)
$$

Where, $E_{D e}$ is dematerialization, $E_{S a}$ is immaterialization (energy saving) and $E_{R e}$ is energy rebound effect

From solved of above matrix we get

$$
E s=\left(\begin{array}{l}
E_{D e} \\
E_{S a} \\
E_{\mathrm{Re}}
\end{array}\right)=\left(\begin{array}{l}
-I_{\text {effect }} \\
-I_{\text {effect }}-S_{\text {effect }} \\
0+S_{\text {effect }}+G D P_{\text {effect }}
\end{array}\right)
$$

Here,

$\mathrm{E}_{\mathrm{De}}=$ Dematerialization $=-\mathrm{I}_{\mathrm{effect}}$

$\mathrm{E}_{\mathrm{Sa}}=$ Immaterialization $=$ Energy saving $=-\left(\mathrm{I}_{\text {effect }}+\mathrm{S}_{\text {effect }}\right)$

$\mathrm{E}_{\mathrm{Re}}=$ Energy Rebound effect $=\mathrm{S}_{\text {effect }}+\mathrm{GDP}_{\text {effect }}$

The equation (16) is used in energy rebound effect calculation.

\section{Results and Discussions}

As the industrial sector consumes the major amount of energy and contributes to the economic development substantially, energy conservation activities have targeted this sector. Energy consumption in this sector during 2007-2030 was 1265.8 MTOE (see Table I). It accounted for $58 \%$ of the total energy consumption. Hence, energy conservation in this sector is vital. Emphasis will be placed on analyzing energy saving in this particular sector.

During the period 2008-2030 we observed that energy saving occurred in agriculture sector, of which -30.04 MTOE of energy saving was due to structural changes $\left(\mathrm{S}_{\text {effect }}\right)$, and 17.42 MTOE of energy saving was due to intensity changes $\left(\mathrm{I}_{\text {effect }}\right)$ as shown in Table II. The agriculture sector, however, failed to save energy in every year. In Table II it is found that trend value is greater than real value, that is, the value of is less than zero (trend of graph is decreasing), which is the condition for energy saving as shown in Figure 1.

Energy saving did not occur in industrial sector as shown in Table III. During the period 2008 to 2030 the extra energy consumption (227 MTOE) in industry sector came from structural change $\left(\mathrm{S}_{\text {effect }}\right)$ and intensity change $\left(\mathrm{I}_{\text {effect }}\right)$ with amounts of 231.7 MTOE and -4.6 MTOE respectively. In Table III it is found that 2007 to 2030 the real value is grater 
Table I: Energy consumption, GDP and energy intensity in Bangladesh (BBS, 1992-2008)

\begin{tabular}{|c|c|c|c|c|c|c|}
\hline Bangladesh & 2007 & 2015 & 2020 & 2025 & 2030 & $2007-2030$ \\
\hline EC (MTOE) & 28.72 & 53.66 & 85.35 & 136.46 & 214.82 & 2188.57 \\
\hline GDP (mill. US\$) & 60412 & 100309.84 & 158612.7 & 255447.4 & 411400.5 & 4137693 \\
\hline I (KGOE/US\$) & 0.4754022 & 0.53 & 0.538 & 0.534 & 0.522 & 0.528 \\
\hline \multicolumn{7}{|l|}{ Agriculture sector } \\
\hline EC (MTOE) & 3.25 & 5.097 & 6.828 & 10.74 & 10.74 & 143 \\
\hline GDP (mill. US\$) & 12478 & 20061.96 & 28550.29 & 35762.63 & 53482 & 639487 \\
\hline I (KGOE/US\$) & 0.26 & 0.254 & 0.239 & 0.2098 & 0.2 & 0.22 \\
\hline \multicolumn{7}{|l|}{ Industry sector } \\
\hline EC (MTOE) & 13.24 & 27.08 & 133.19 & 81.19 & 133.19 & 1265.8 \\
\hline GDP (mill. US\$) & 17192 & 32099 & 57100 & 109842 & 185130 & 1669264 \\
\hline I (KGOE/US\$) & 0.76 & 0.84 & 083 & 0.73 & 0.71 & 0.75 \\
\hline \multicolumn{7}{|l|}{ Service sector } \\
\hline EC (MTOE) & 12.21 & 21.46 & 70.89 & 46.39 & 70.89 & 774.8 \\
\hline GDP (mill. US\$) & 30742 & 48148 & 172788 & 109842 & 172788 & 1828941 \\
\hline I (KGOE/US\$) & 0.42 & 0.44 & 0.41 & 0.42 & 0.41 & 0.42 \\
\hline
\end{tabular}

Table II: Energy saving in agriculture sector

\begin{tabular}{lccccccc}
\hline Year & $\begin{array}{c}\text { Activity } \\
\text { Effect (MTOE) }\end{array}$ & $\begin{array}{c}\text { Structural } \\
\text { Effect (MTOE) }\end{array}$ & $\begin{array}{c}\text { Intensity } \\
\text { Effect (MTOE) }\end{array}$ & $\begin{array}{c}\text { Del Eot } \\
\text { (MTOE) }\end{array}$ & $\begin{array}{c}\text { Real Energy.. } \\
\text { consm (MTOE) }\end{array}$ & $\begin{array}{c}\text { Trend energy. } \\
\text { consm (MTOE) }\end{array}$ & $\begin{array}{c}\text { Energy saving } \\
\text { (MTOE) }\end{array}$ \\
\hline 2008 & 0.168 & 0.211 & -0.216 & 0.163 & 28.883 & 28.88 & -0.0058 \\
2009 & 0.350 & 0.219 & -0.134 & 0.435 & 29.155 & 29.07 & 0.0849 \\
2010 & 0.559 & 0.059 & -0.080 & 0.538 & 29.258 & 29.27 & -0.0211 \\
2011 & 0.793 & 0.061 & -0.025 & 0.830 & 29.551 & 29.51 & 0.0364 \\
2012 & 1.045 & 0.064 & 0.034 & 1.144 & 29.864 & 29.76 & 0.0986 \\
2013 & 1.328 & -0.121 & -0.138 & 1.068 & 29.788 & 30.048 & -0.259 \\
2014 & 1.690 & -0.127 & -0.121 & 1.441 & 30.161 & 30.41 & -0.2488 \\
2015 & 2.083 & -0.134 & -0.103 & 1.846 & 30.566 & 30.803 & -0.2368 \\
2016 & 2.509 & -0.141 & -0.082 & 2.286 & 31.006 & 31.229 & -0.223 \\
2017 & 2.834 & -0.590 & -0.365 & 1.878 & 30.598 & 31.554 & -0.956 \\
2018 & 3.409 & -0.629 & -0.389 & 2.390 & 31.110 & 32.129 & -1.0191 \\
2019 & 4.042 & -0.672 & -0.415 & 2.953 & 31.673 & 32.76 & -1.0881 \\
2020 & 4.737 & -0.719 & -0.445 & 3.573 & 32.293 & 33.457 & -1.164 \\
2021 & 4.851 & -1.551 & -0.921 & 2.379 & 31.099 & 33.57 & -2.472 \\
2022 & 5.397 & -1.938 & -1.043 & 2.415 & 31.135 & 34.12 & -2.982 \\
2023 & 6.180 & -2.081 & -1.129 & 2.970 & 31.690 & 34.90 & -3.210 \\
2024 & 7.041 & -2.238 & -1.223 & 3.578 & 32.298 & 35.76 & -3.462 \\
2025 & 7.986 & -2.4106 & -1.328 & 4.247 & 32.967 & 36.706 & -3.738 \\
2026 & 8.691 & -2.952 & -1.526 & 4.213 & 32.933 & 37.411 & -4.4779 \\
2027 & 9.780 & -3.183 & -1.675 & 4.921 & 33.641 & 38.500 & -4.8588 \\
2028 & 10.97 & -3.437 & -1.842 & 5.697 & 34.417 & 39.695 & -5.278 \\
2029 & 12.288 & -3.715 & -2.026 & 6.546 & 35.266 & 41.008 & -5.7417 \\
2030 & 13.728 & -4.021 & -2.231 & 7.476 & 36.196 & 42.448 & -6.2523 \\
$2008-30$ & 112.47 & -30.049 & -17.42 & 64.99 & 725.55 & 773.03 & -47.477 \\
\hline
\end{tabular}

than trend value which is an unsatisfactory condition of energy saving. Punyong 2008, stated that the energy saving in Thai industry was 1401.95 KTOE (over consumption instead of saving) during the period 1998 to 2002.
Energy consumption in service sector during the period 2007 to 2030 was 774.8 MTOE (see Table I). It accounted for $35.4 \%$ of the total energy consumption. During the period 2008-2030 we observed that energy saving occurred in service sector, of which -71.68 MTOE of energy saving was due 


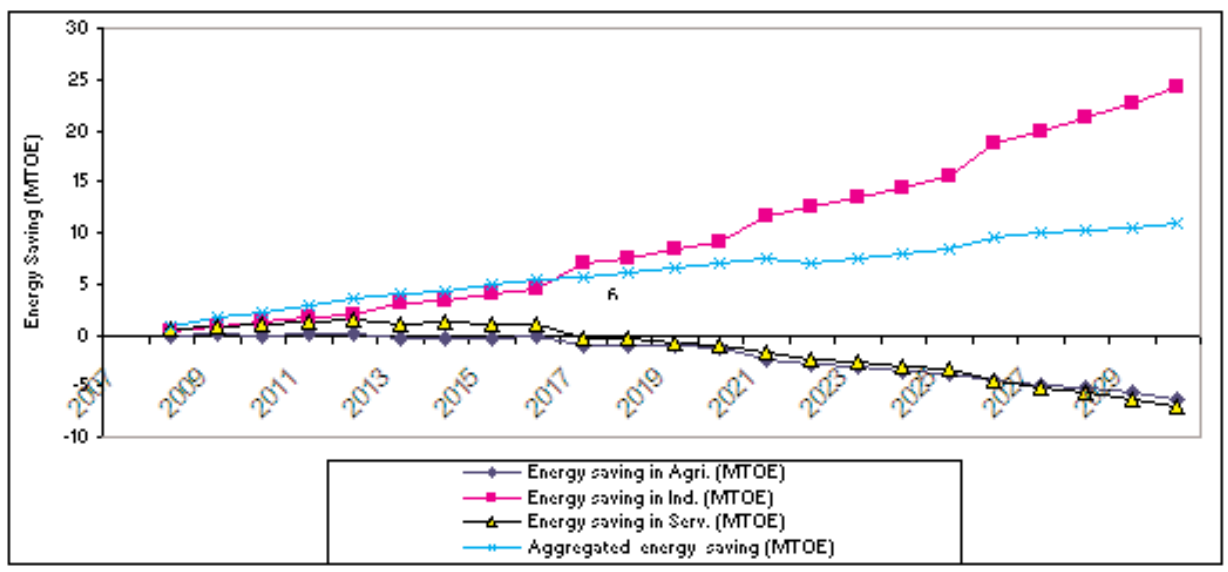

Fig. 1: Sector wise energy saving

to structural changes $\left(\mathrm{S}_{\text {effect }}\right)$, and extra energy (36.72 MTOE) came from intensity changes (Ieffect) as shown in Table IV. In Table IV it is found that trend value is greater than real value, that is, the value of $\psi$ is less than zero (trend of graph is decreasing), which is the condition for energy saving as shown in Figure 1.

The analysis shows that, during the period 2008-2030, the total energy saving indicator in Bangladesh was 144.66 MTOE (positive value means over-consumption instead of saving). This indicator when resolved into three sectors namely agriculture, industry and service respectively, their corresponding values turns out to be -47.47 MTOE, 227.10 MTOE and -34.96 MTOE respectively. Since the energy consumed by the agriculture sector was only $9.8 \%$ of the total energy consumption, its contribution to the energy saving is minimal. In our previous analysis it was found that during the period of 1990 to 2007 only agriculture saves energy, on the other hand industry and service sectore failed to save energy. During the period 2008 to 2030 the extra-

Table III: Energy saving in Industrial sector

\begin{tabular}{|c|c|c|c|c|c|c|c|}
\hline Year & $\begin{array}{c}\text { Activity } \\
\text { Effect (MTOE) }\end{array}$ & $\begin{array}{c}\text { Structural } \\
\text { Effect (MTOE) }\end{array}$ & $\begin{array}{c}\text { Intensity } \\
\text { Effect (MTOE) }\end{array}$ & $\begin{array}{l}\text { Del Eot } \\
\text { (MTOE) }\end{array}$ & $\begin{array}{l}\text { Real Energy.. } \\
\text { consm (MTOE) }\end{array}$ & $\begin{array}{l}\text { Trend energy. } \\
\text { consm (MTOE) }\end{array}$ & $\begin{array}{c}\text { Energy saving } \\
\text { (MTOE) }\end{array}$ \\
\hline 2008 & 0.697 & -0.219 & 0.555 & 1.032 & 29.752 & 29.416 & 0.335 \\
\hline 2009 & 1.449 & -0.228 & 0.953 & 2.174 & 30.893 & 30.169 & 0.724 \\
\hline 2010 & 2.396 & 0.797 & 0.527 & 3.721 & 32.440 & 31.115 & 1.325 \\
\hline 2011 & 3.398 & 0.831 & 0.797 & 5.026 & 33.746 & 32.117 & 1.628 \\
\hline 2012 & 4.476 & 0.866 & 1.091 & 6.433 & 35.152 & 33.196 & 1.957 \\
\hline 2013 & 6.144 & 2.066 & 1.058 & 9.268 & 37.988 & 34.864 & 3.125 \\
\hline 2014 & 7.819 & 2.172 & 1.221 & 11.21 & 39.933 & 36.539 & 3.393 \\
\hline 2015 & 9.7423 & 2.311 & 1.808 & 13.86 & 42.582 & 38.4623 & 4.120 \\
\hline 2016 & 11.738 & 2.436 & 2.028 & 16.20 & 44.923 & 40.458 & 4.465 \\
\hline 2017 & 15.093 & 5.416 & 1.524 & 22.03 & 50.753 & 43.813 & 6.940 \\
\hline 2018 & 18.154 & 5.778 & 1.627 & 25.56 & 54.280 & 46.874 & 7.406 \\
\hline 2019 & 21.731 & 6.242 & 2.243 & 30.22 & 58.935 & 50.451 & 8.485 \\
\hline 2020 & 25.471 & 6.685 & 2.404 & 34.56 & 63.281 & 54.191 & 9.089 \\
\hline 2021 & 30.549 & 10.632 & 0.975 & 42.15 & 70.876 & 59.269 & 11.607 \\
\hline 2022 & 35.507 & 13.847 & -1.254 & 48.10 & 76.821 & 64.227 & 12.594 \\
\hline 2023 & 40.649 & 14.882 & -1.419 & 54.11 & 82.832 & 69.369 & 13.462 \\
\hline 2024 & 46.30 & 16.018 & -1.606 & 60.71 & 89.432 & 75.020 & 14.412 \\
\hline 2025 & 52.507 & 17.267 & -1.816 & 67.95 & 96.678 & 81.227 & 15.45 \\
\hline 2026 & 60.754 & 21.114 & -2.460 & 79.40 & 108.128 & 89.47 & 18.653 \\
\hline 2027 & 68.330 & 22.784 & -2.905 & 88.21 & 116.929 & 97.05 & 19.878 \\
\hline 2028 & 76.640 & 24.616 & -3.410 & 97.85 & 126.566 & 105.36 & 21.206 \\
\hline 2029 & 85.756 & 26.627 & -3.984 & 108.3 & 137.119 & 114.476 & 22.643 \\
\hline 2030 & 95.756 & 28.832 & -4.634 & 119.9 & 148.675 & 124.476 & 24.198 \\
\hline $2008-30$ & 721.061 & 231.777 & -4.676 & 948.2 & 1608.722 & 1381.62 & 227.101 \\
\hline
\end{tabular}


Table IV: Energy saving in Service sector

\begin{tabular}{lccccccc}
\hline Year & $\begin{array}{c}\text { Activity } \\
\text { Effect (MTOE) }\end{array}$ & $\begin{array}{c}\text { Structural } \\
\text { Effect (MTOE) }\end{array}$ & $\begin{array}{c}\text { Intensity } \\
\text { Effect (MTOE) }\end{array}$ & $\begin{array}{c}\text { Del Eot } \\
\text { (MTOE) }\end{array}$ & $\begin{array}{c}\text { Real Energy.. } \\
\text { consm (MTOE) }\end{array}$ & $\begin{array}{c}\text { Trend energy. } \\
\text { consm (MTOE) }\end{array}$ & $\begin{array}{c}\text { Energy saving } \\
\text { (MTOE) }\end{array}$ \\
\hline 2008 & 0.647 & -0.223 & 0.711 & 1.134 & 29.604 & 29.367 & 0.487 \\
2009 & 1.346 & -0.232 & 1.0879 & 2.202 & 30.672 & 30.065 & 0.856 \\
2010 & 2.189 & -0.518 & 1.459 & 3.130 & 31.600 & 30.908 & 0.942 \\
2011 & 3.105 & -0.539 & 1.746 & 4.311 & 32.781 & 31.825 & 1.206 \\
2012 & 4.091 & -0.563 & 2.055 & 5.583 & 34.053 & 32.811 & 1.492 \\
2013 & 5.364 & -0.897 & 1.972 & 6.437 & 34.907 & 34.084 & 1.0734 \\
2014 & 6.827 & -0.944 & 2.164 & 8.047 & 36.517 & 35.547 & 1.220 \\
2015 & 8.307 & -0.979 & 1.933 & 9.261 & 37.731 & 37.027 & 0.953 \\
2016 & 10.009 & -1.033 & 2.139 & 11.115 & 39.585 & 38.729 & 1.106 \\
2017 & 11.800 & -1.826 & 1.549 & 11.523 & 39.994 & 40.521 & -0.277 \\
2018 & 14.194 & -1.948 & 1.651 & 13.896 & 42.366 & 42.914 & -0.297 \\
2019 & 16.593 & -2.050 & 1.187 & 15.730 & 44.200 & 45.313 & -0.863 \\
2020 & 19.448 & -2.195 & 1.270 & 18.523 & 46.994 & 48.168 & -0.925 \\
2021 & 22.285 & -2.827 & 1.200 & 20.657 & 49.127 & 51.005 & -1.627 \\
2022 & 25.338 & -4.108 & 1.617 & 22.846 & 51.316 & 54.058 & -2.492 \\
2023 & 29.009 & -4.417 & 1.689 & 26.282 & 54.752 & 57.729 & -2.728 \\
2024 & 33.043 & -4.757 & 1.767 & 30.053 & 58.523 & 61.763 & -2.990 \\
2025 & 37.475 & -5.131 & 1.850 & 34.194 & 62.664 & 66.195 & -3.281 \\
2026 & 41.700 & -6.209 & 1.616 & 37.107 & 65.577 & 70.420 & -4.593 \\
2027 & 46.906 & -6.703 & 1.589 & 41.792 & 70.262 & 75.626 & -5.114 \\
2028 & 52.617 & -7.244 & 1.548 & 46.92 & 75.391 & 81.337 & -5.695 \\
2029 & 58.884 & -7.838 & 1.493 & 52.538 & 81.008 & 87.604 & -6.345 \\
2030 & 65.758 & -8.490 & 1.420 & 58.688 & 87.159 & 94.478 & -7.069 \\
$2008-30$ & 516.939 & -71.682 & 36.720 & 481.97 & 1136.787 & 1177.499 & -34.962 \\
\hline
\end{tabular}

Table V: Aggregated energy saving

\begin{tabular}{lcccc}
\hline Year & $\begin{array}{c}\text { Energy saving in agri. } \\
\text { (MTOE) }\end{array}$ & $\begin{array}{c}\text { Energy saving in industry. } \\
\text { (MTOE) }\end{array}$ & $\begin{array}{c}\text { Energy saving in service } \\
\text { (MTOE) }\end{array}$ & $\begin{array}{c}\text { Aggregate energy saving } \\
\text { (MTOE) }\end{array}$ \\
\hline 2008 & -0.005 & 0.335 & 0.487 & 0.817 \\
2009 & 0.0849 & 0.724 & 0.855 & 1.665 \\
2010 & -0.0211 & 1.325 & 0.942 & 2.245 \\
2011 & 0.036 & 1.628 & 1.206 & 2.871 \\
2012 & 0.098 & 1.956 & 1.492 & 3.547 \\
2013 & -0.259 & 3.125 & 1.073 & 3.939 \\
2014 & -0.248 & 3.393 & 1.220 & 4.365 \\
2015 & -0.236 & 4.120 & 0.953 & 4.836 \\
2016 & -0.223 & 4.464 & 1.106 & 5.347 \\
2017 & -0.956 & 6.940 & -0.276 & 5.707 \\
2018 & -1.019 & 7.406 & -0.297 & 6.089 \\
2019 & -1.088 & 8.4847 & -0.863 & 6.533 \\
2020 & -1.164 & 9.089 & -0.925 & 7.001 \\
2021 & -2.472 & 11.607 & -1.627 & 7.507 \\
2022 & -2.982 & 12.594 & -2.492 & 7.121 \\
2023 & -3.210 & 13.462 & -2.728 & 7.524 \\
2024 & -3.462 & 14.412 & -2.990 & 7.960 \\
2025 & -3.738 & 15.4509 & -3.281 & 8.431 \\
2026 & -4.478 & 18.654 & -4.593 & 9.583 \\
2027 & -4.859 & 19.878 & -5.114 & 9.906 \\
2028 & -5.279 & 21.206 & -5.695 & 10.231 \\
2029 & -5.742 & 22.643 & -6.345 & 10.556 \\
2030 & -6.252 & 24.198 & -7.069 & 10.876 \\
$2008-30$ & -47.477 & 227.101 & -34.962 & 144.66 \\
\hline
\end{tabular}


energy consumption (144.66 MTOE) in Bangladesh came from Structural change $\left(\mathrm{S}_{\text {effect }}\right)$ and intensity changes $\left(\mathrm{I}_{\text {effect }}\right)$ have values 130.05 MTOE and 14.62 MTOE respectively.

The energy rebound effect, which is the combined result of activity effect and structural effect, is found to increase in agriculture, industry and service sector as shown in Figure 2.
In agriculture sector rebound effect increased by 25.6 fold in 2030, compared to that in 2008 (Table VI). Again, rebound effect increased by 262 fold and 135 fold in industry and service sector respectively in 2030 compared to 2008 . The aggregate rebound effects increased by 149.5 fold in the timeperiod of 2008 to 2030 of which activity effect contributes $1350.46 \mathrm{MTOE}$ and structural effect contributes

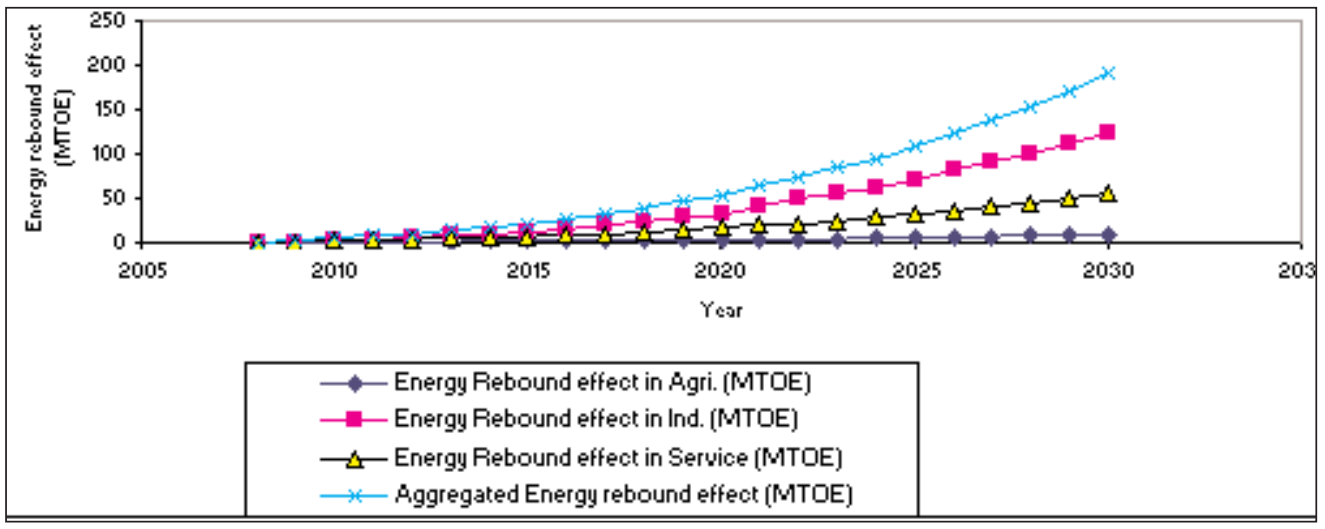

Fig. 2: sector wise energy rebound effect

Table VI: Aggregate energy rebound effect

\begin{tabular}{|c|c|c|c|c|}
\hline Year & $\begin{array}{l}\text { Energy rebound } \\
\text { effect in Agri. } \\
\text { (MTOE) }\end{array}$ & $\begin{array}{l}\text { Energy rebound } \\
\text { effect in Ind. } \\
\text { (MTOE) }\end{array}$ & $\begin{array}{c}\text { Energy rebound } \\
\text { effect in Service } \\
(\mathrm{MTOE})\end{array}$ & $\begin{array}{c}\text { Aggregated } \\
\text { Energy rebound effect } \\
(\mathrm{MTOE})\end{array}$ \\
\hline 2008 & 0.379 & 0.477 & 0.4242 & 1.281 \\
\hline 2009 & 0.569 & 1.221 & 1.114 & 2.904 \\
\hline 2010 & 0.618 & 3.194 & 1.671 & 5.483 \\
\hline 2011 & 0.855 & 4.228 & 2.565 & 7.648 \\
\hline 2012 & 1.1093 & 5.342 & 3.527 & 9.978 \\
\hline 2013 & 1.2067 & 8.2106 & 4.466 & 13.883 \\
\hline 2014 & 1.563 & 9.991 & 5.883 & 17.438 \\
\hline 2015 & 1.949 & 12.053 & 7.327 & 21.330 \\
\hline 2016 & 2.368 & 14.175 & 8.976 & 25.519 \\
\hline 2017 & 2.244 & 20.508 & 9.9738 & 32.726 \\
\hline 2018 & 2.779 & 23.932 & 12.245 & 38.956 \\
\hline 2019 & 3.369 & 27.973 & 14.543 & 45.885 \\
\hline 2020 & 4.0180 & 32.156 & 17.253 & 53.427 \\
\hline 2021 & 3.300 & 41.181 & 19.457 & 63.939 \\
\hline 2022 & 3.4589 & 49.354 & 21.229 & 74.043 \\
\hline 2023 & 4.099 & 55.532 & 24.592 & 84.222 \\
\hline 2024 & 4.802 & 62.318 & 28.285 & 95.406 \\
\hline 2025 & 5.5756 & 69.775 & 32.344 & 107.695 \\
\hline 2026 & 5.7392 & 81.868 & 35.490 & 123.098 \\
\hline 2027 & 6.597 & 91.114 & 40.203 & 137.914 \\
\hline 2028 & 7.5386 & 101.256 & 45.373 & 154.168 \\
\hline 2029 & 8.5723 & 112.383 & 51.045 & 172.000 \\
\hline 2030 & 9.707 & 124.588 & 57.268 & 191.564 \\
\hline $2008-2030$ & 82.420 & 952.838 & 445.257 & 1480.515 \\
\hline
\end{tabular}


129.98 MTOE respectively. From rebound effect analysis it is found that the technological development has increased in industry and service sector rather than agriculture sector and our structure of economy is shifting from agriculture to industry, but with no good effect in respect of energy saving. The reason is that there have been more structural changes than new innovations in industries.

\section{Conclusion}

This paper presents a detailed analysis of energy saving and energy rebound effect in Bangladesh. It can be concluded that,

1) In the time period of 2008 to 2030, energy saving occurred in agriculture sector of an amount -47.47 MTOE.

2) Energy saving did not happen in industrial sector. Extra energy consumption (227 MTOE) in industry sector came from structural change $\left(\mathrm{S}_{\text {effect }}\right)$ and intensity change ( $\left.\mathrm{I}_{\text {effect }}\right)$ with amount of 231.7 MTOE and 4.6 MTOE respectively.

3) Energy saving occurred in service sector. During the period 2008 to 2030 the energy consumption $(-34.96$ MTOE) in service sector came from structural change $\left(\mathrm{S}_{\text {effect }}\right)$ and intensity change $\left(\mathrm{I}_{\text {effect }}\right)$ with amount of 71.68 MTOE and 36.72 MTOE respectively.

4) The aggregate energy saving in Bangladesh was +144.66 MTOE in the time period of 2008 to 2030 . The positive value indicates the over-consumption instead of saving which is the general characteristic of infrastructure building period.

5) The aggregated rebound effects increased by 149.5 fold in the time period of 2008 to 2030 of which activity effect contributes 1350.46 MTOE and structural effect contributes 129.98 MTOE respectively. From rebound effect analysis it is found that the technological development has increased in industry and service sector rather than agriculture sector and our structure of economy is shifting from agriculture to industry, but with no good effect in respect of energy saving. The reason is that there have been more structural changes than new innovations in industries.
It appears that as in most developing countries there has been more stress on administrative measures for structural changes than scientific and technological innovations in industries, which we consider to be the main barriers to energy saving through greater efficiency.

\section{References}

Ang, BW and Zhang FQ (2000). A survey of index decomposition analysis in energy and environmental studies, Energy, 25: 1149-1176.

Bangladesh Bureau of Statistics (BBS). 1992-2008. Statistical pocketbook of Bangladesh. Ministry of Finance and Planning, Govt. of the People's Republic of. Bangladesh, Dhaka.

Malaska P, Luukkanen J and Kaivo-oja J (1999). Decomposition method in sustainability analysis. FUTUPublications 3/99. Turku School of Economics and Business Administration. Finland Futures Research Centre. Turku.

Punyong K, Taweekun J and Prasertsan S (2008). Evaluation of energy saving in Thai industry by 3-D decomposition method. Asian J. Energy Environ. 9(1 \&2): 15-37.

Sun JW (2003). Dematerialization in Finnish energy use, 1972-1996, Energy Economics, 25: 23-32.

Sun JW (2001). Energy demand in the fifteen European Union countries by 2010 a forecasting model based on the decomposition approach. Energy, 26: 549-60.

Sun JW (1998). Change in energy consumption and energy strength: A complete decomposition model, Energy Econ. 20: 85-100.

Received : 17 May 2010; Revised : 14 June 2010; Accepted: 25 April 2011 